\title{
COX RINGS OF EXTREMAL RATIONAL ELLIPTIC SURFACES
}

\author{
MICHELA ARTEBANI, ALICE GARBAGNATI, AND ANTONIO LAFACE \\ Abstract. In this paper we determine a minimal set of generators for the Cox \\ rings of extremal rational elliptic surfaces. Moreover, we develop a technique \\ for computing the ideal of relations between them which allows us to provide \\ a presentation of the Cox ring in most cases.
}

\section{INTRODUCTION}

Let $X$ be a smooth projective rational surface over $\mathbb{C}$ and $\pi: X \rightarrow \mathbb{P}^{1}$ be a relatively minimal jacobian elliptic fibration on $X$. In this paper we are interested in the Cox ring of $X$, which is the $\operatorname{Pic}(X)$-graded ring defined as follows (see Section 1):

$$
\mathcal{R}(X):=\bigoplus_{[D] \in \operatorname{Pic}(X)} H^{0}\left(X, \mathcal{O}_{X}(D)\right)
$$

By AL11 the Cox ring $\mathcal{R}(X)$ is a finitely generated algebra if and only if the Mordell-Weil group of $\pi$ is finite, which means that $\pi$ admits just a finite number of sections. The elliptic fibrations with such property are called extremal, after the work of Miranda and Persson who classified them in [MP86]. The aim of this paper is to give an explicit presentation for the Cox rings of such surfaces. Our main theorem is the following.

Theorem. Let $X$ be an extremal rational elliptic surface. Then its Cox ring is generated by sections in the following degrees:

(i) the anticanonical class $-K_{X}$;

(ii) classes of smooth irreducible rational curves $C$ with $C^{2} \in\{-2,-1,0,1\}$.

We now give a short description of the content of the paper. Section 1 recalls the basic properties of elliptic fibrations and some preliminary results about Cox rings. In Section 2 we prove the main theorem, while Section 3 is devoted to elliptic surfaces of complexity one, that is, admitting an effective $\mathbb{C}^{*}$-action. The Cox ring of such surfaces is computed by means of a technique developed in [Hau13, §3.3]. In Section 4 we provide a method for computing the ideal of relations of the Cox ring. This allows us to compute explicitly the Cox ring of all but three extremal rational elliptic surfaces. Finally, Section 5 deals with some applications of the previous results, as computing the Cox ring of the Cayley cubic and that of weak del Pezzo surfaces which are dominated by extremal rational elliptic surfaces.

Received by the editors May 1, 2013 and, in revised form, January 1, 2014.

2010 Mathematics Subject Classification. Primary 14J26, 14C20.

Key words and phrases. Cox rings, rational elliptic surfaces.

The first author was partially supported by Proyecto FONDECYT Regular N. 1110249 and N. 1130572. The second author was partially supported by PRIN 2010-2011 "Geometria delle varietà algebriche" and FIRB 2012 "Moduli Spaces and their Applications". The third author was partially supported by Proyecto FONDECYT regular N. 1110096 and N. 1150732. 


\section{BASIC SETUP}

1.1. Elliptic surfaces. We briefly recall some well-known facts about elliptic fibrations. A good and recent reference for these results is [SS10].

Let $X$ be a smooth projective surface over $\mathbb{C}$ and $\pi: X \rightarrow \mathbb{P}^{1}$ be an elliptic fibration, that is, a morphism whose general fiber is a smooth curve of genus one. In what follows we will assume $\pi$ to be jacobian, i.e., $\pi$ admits a section, and relatively minimal, i.e., $\pi$ does not contract $(-1)$-curves. Given a zero section $P_{0}$, the set of sections of $\pi$ forms a finitely generated abelian group, the Mordell-Weil group of $\pi$, which will be denoted by $M W(\pi)$.

The singular fibers of an elliptic fibration have been classified by Kodaira Kod63. according to the types $\mathrm{I}_{n}(n \geq 1)$, II, III, IV, II*, III*, IV*, $\mathrm{I}_{n}^{*}(n \geq 0)$. The intersection graph of some of the reducible fibers is represented in Figure 1) where each vertex represents a $(-2)$-curve and the number near to it is its multiplicity.

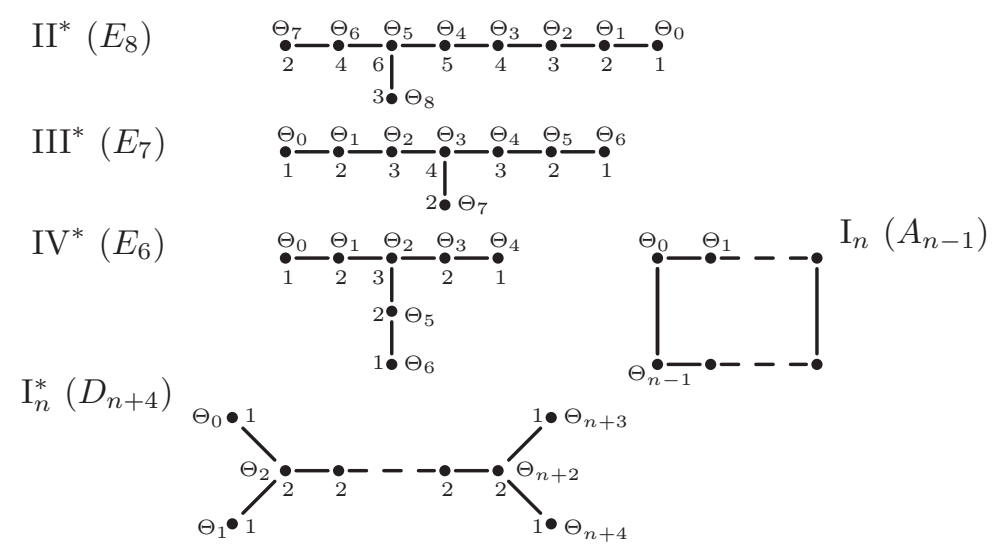

Figure 1. Some reducible fibers of an elliptic fibration

Rational elliptic surfaces with finite Mordell-Weil group have been classified by Miranda and Persson in MP86. Their result is summarized in Table 1.

TABLE 1. Extremal rational elliptic surfaces

\begin{tabular}{l|l|l||l|l|l} 
Surface & Type & MW $(\pi)$ & Surface & Type & $\mathrm{MW}(\pi)$ \\
\hline$X_{22}$ & $\mathrm{II}^{*} \mathrm{II}$ & $\{0\}$ & $X_{431}$ & $\mathrm{IV}^{*} \mathrm{I}_{3} \mathrm{I}_{1}$ & $\mathbb{Z} / 3 \mathbb{Z}$ \\
$X_{211}$ & $\mathrm{II}^{*} 2 \mathrm{I}_{1}$ & $\{0\}$ & $X_{222}$ & $\mathrm{I}_{2}^{*} 2 \mathrm{I}_{2}$ & $(\mathbb{Z} / 2 \mathbb{Z})^{\oplus 2}$ \\
$X_{411}$ & $\mathrm{I}_{4}^{*} 2 \mathrm{I}_{1}$ & $\mathbb{Z} / 2 \mathbb{Z}$ & $X_{141}$ & $\mathrm{I}_{1}^{*} \mathrm{I}_{4} \mathrm{I}_{1}$ & $\mathbb{Z} / 4 \mathbb{Z}$ \\
$X_{9111}$ & $\mathrm{I}_{9} 3 \mathrm{I}_{1}$ & $\mathbb{Z} / 3 \mathbb{Z}$ & $X_{6321}$ & $\mathrm{I}_{6} \mathrm{I}_{3} \mathrm{I}_{2} \mathrm{I}_{1}$ & $\mathbb{Z} / 6 \mathbb{Z}$ \\
$X_{33}$ & III $^{*} \mathrm{III}$ & $\mathbb{Z} / 2 \mathbb{Z}$ & $X_{11}(a)$ & $2 \mathrm{I}_{0}^{*}$ & $(\mathbb{Z} / 2 \mathbb{Z})^{\oplus 2}$ \\
$X_{321}$ & $\mathrm{III}^{*} \mathrm{I}_{2} \mathrm{I}_{1}$ & $\mathbb{Z} / 2 \mathbb{Z}$ & $X_{5511}$ & $2 \mathrm{I}_{5} 2 \mathrm{I}_{1}$ & $\mathbb{Z} / 5 \mathbb{Z}$ \\
$X_{8211}$ & $\mathrm{I}_{8} \mathrm{I}_{2} 2 \mathrm{I}_{1}$ & $\mathbb{Z} / 4 \mathbb{Z}$ & $X_{4422}$ & $2 \mathrm{I}_{4} 2 \mathrm{I}_{2}$ & $\mathbb{Z} / 4 \mathbb{Z} \oplus \mathbb{Z} / 2 \mathbb{Z}$ \\
$X_{44}$ & $\mathrm{IV}^{*} \mathrm{IV}$ & $\mathbb{Z} / 3 \mathbb{Z}$ & $X_{3333}$ & $4 \mathrm{I}_{3}$ & $(\mathbb{Z} / 3 \mathbb{Z})^{\oplus 2}$
\end{tabular}


A smooth projective rational surface with a jacobian elliptic fibration $\pi: X \rightarrow \mathbb{P}^{1}$ is known to be isomorphic to the blow-up of the projective plane at nine points, eventually infinitely near. In fact, $X$ carries a unique elliptic fibration, induced by the pencil of cubics in $\mathbb{P}^{2}$ through the nine points. Since the class of a fiber of $\pi$ is the anticanonical class, the sections of $\pi$ are exactly the $(-1)$-curves. In Table 2 we give the equation of a pencil of cubic curves giving rise to any such surface.

TABLE 2. Pencils of cubics inducing extremal elliptic surfaces

\begin{tabular}{l|l} 
Surface & Pencil of cubics \\
\hline$X_{22}$ & $\left(x_{1}^{3}+x_{0}^{2} x_{2}\right)+t x_{2}^{3}=0$ \\
$X_{211}$ & $\left(x_{1}^{3}+x_{0}^{2} x_{2}+x_{1}^{2} x_{2}\right)+t x_{2}^{3}=0$ \\
$X_{411}$ & $\left(x_{0}^{2} x_{1}+x_{2}^{3}+x_{1}^{2} x_{2}\right)+t x_{1} x_{2}^{2}=0$ \\
$X_{9111}$ & $\left(x_{0}^{2} x_{1}+x_{1}^{2} x_{2}+x_{2}^{2} x_{0}\right)+t x_{0} x_{1} x_{2}=0$ \\
$X_{33}$ & $x_{0}\left(x_{0} x_{2}-x_{1}^{2}\right)+t x_{2}^{3}=0$ \\
$X_{321}$ & $x_{0}\left(x_{0} x_{2}-x_{1}^{2}+x_{1} x_{2}\right)+t x_{2}^{3}=0$ \\
$X_{8211}$ & $\left(x_{0}-x_{1}\right)\left(x_{0} x_{1}-x_{2}^{2}\right)+t x_{0} x_{1} x_{2}=0$ \\
$X_{44}$ & $x_{1} x_{2}\left(x_{1}-x_{2}\right)+t x_{0}^{3}=0$ \\
$X_{431}$ & $x_{1} x_{2}\left(x_{0}+x_{1}+x_{2}\right)+t x_{0}^{3}=0$ \\
$X_{222}$ & $x_{0} x_{1}\left(x_{0}-x_{1}\right)+t\left(x_{1}^{3}+2 x_{0} x_{1} x_{2}-2 x_{1}^{2} x_{2}-x_{0} x_{2}^{2}+x_{1} x_{2}^{2}\right)=0$ \\
$X_{141}$ & $x_{2}\left(x_{0} x_{1}-x_{1}^{2}+x_{0} x_{2}\right)+t x_{0} x_{1}\left(x_{0}-x_{1}\right)=0$ \\
$X_{6321}$ & $\left(x_{0}+x_{1}\right)\left(x_{1}+x_{2}\right)\left(x_{0}+x_{2}\right)+t x_{0} x_{1} x_{2}=0$ \\
$X_{11}(a)$ & $x_{1} x_{2}\left(x_{1}-x_{2}\right)+t\left(x_{1}-a x_{2}\right) x_{0}^{2}=0, a \in \mathbb{C}-\{0,1\}$ \\
$X_{5511}$ & $\left(x_{1}+x_{2}\right)\left(x_{0}+x_{1}\right)\left(x_{0}+x_{1}+x_{2}\right)+t x_{0} x_{1} x_{2}=0$ \\
$X_{4422}$ & $\left(x_{0}-x_{2}\right)\left(x_{0}-2 x_{1}+x_{2}\right)\left(x_{0}+2 x_{1}+x_{2}\right)+t x_{0} x_{1} x_{2}=0$ \\
$X_{3333}$ & $\left(x_{0}^{3}+x_{1}^{3}+x_{2}^{3}\right)+t x_{0} x_{1} x_{2}=0$
\end{tabular}

In what follows, it will be useful to know the intersection matrix for the curves with negative intersection on $X$. We recall that, in case $M W(\pi)$ is finite, two sections of $\pi$ do not intersect by [Mir89, Proposition VII.3.2.]. The intersections between the torsion sections and the reducible fibers of extremal rational elliptic surfaces, which can be computed by means of the height formula [Shi90], are summarized in Table 3 .

We will denote by $\Theta_{i}^{j}$ the $i$-th component, with the notation in Figure1, of the $j$ th reducible fiber (once a numbering of the reducible fibers is chosen). In particular $\Theta_{0}^{j}$ will denote the component intersecting the zero section. If the Mordell-Weil group is isomorphic to $\mathbb{Z} / n \mathbb{Z} \oplus \mathbb{Z} / m \mathbb{Z}, n \geq m$, we denote by $P_{1}$ a generator of $\mathbb{Z} / n \mathbb{Z}$ and by $Q_{1}$ a generator of $\mathbb{Z} / m \mathbb{Z}$. Moreover, $P_{j}$ denotes the section which is $j$ times $P_{1}$ with respect to the group law of the Mordell-Weil group. Observe that Table 3 gives the intersection numbers for $P_{1}$ and $Q_{1}$; the remaining intersection numbers can be deduced from these according to the group law. For example in the case $X_{3333}$ the section corresponding to $P_{i}+Q_{j}$ intersects the $n$-th fiber in the component $\Theta_{a i+b j(\bmod 3)}^{n}$, where $P_{1} \cdot \Theta_{a}^{n}=1$ and $Q_{1} \cdot \Theta_{b}^{n}=1$.

1.2. Cox rings. The Cox ring of a complete normal variety with finitely generated and free class group $\mathrm{Cl}(X)$ can be defined as follows:

$$
\mathcal{R}(X):=\bigoplus_{D \in K} H^{0}\left(X, \mathcal{O}_{X}(D)\right)
$$


TABLE 3. Intersections between sections and reducible fibers

\begin{tabular}{c|c|c} 
Surface & $M W(\pi)$ & Intersection properties \\
\hline$X_{411}$ & $\mathbb{Z} / 2 \mathbb{Z}$ & $P_{1} \cdot \Theta_{8}=1$ \\
\hline$X_{9111}$ & $\mathbb{Z} / 3 \mathbb{Z}$ & $P_{1} \cdot \Theta_{3}=1$ \\
\hline$X_{33}, X_{321}$ & $\mathbb{Z} / 2 \mathbb{Z}$ & $P_{1} \cdot \Theta_{6}^{1}=P_{1} \cdot \Theta_{1}^{2}=1$ \\
\hline$X_{8211}$ & $\mathbb{Z} / 4 \mathbb{Z}$ & $P_{1} \cdot \Theta_{2}^{1}=P_{1} \cdot \Theta_{1}^{2}=1$ \\
\hline$X_{44}, X_{431}$ & $\mathbb{Z} / 3 \mathbb{Z}$ & $P_{1} \cdot \Theta_{6}^{1}=P_{1} \cdot \Theta_{1}^{2}=1$ \\
\hline$X_{222}$ & $(\mathbb{Z} / 2 \mathbb{Z})^{\oplus 2}$ & $P_{1} \cdot \Theta_{1}^{1}=P_{1} \cdot \Theta_{1}^{2}=P_{1} \cdot \Theta_{1}^{3}=1$ \\
& & $Q_{1} \cdot \Theta_{6}^{1}=Q_{1} \cdot \Theta_{1}^{1}=Q_{1} \cdot \Theta_{0}^{3}=1$ \\
\hline$X_{141}$ & $\mathbb{Z} / 4 \mathbb{Z}$ & $P_{1} \cdot \Theta_{5}^{1}=P_{1} \cdot \Theta_{1}^{2}=1$ \\
\hline$X_{6321}$ & $\mathbb{Z} / 6 \mathbb{Z}$ & $P_{1} \cdot \Theta_{1}^{i}=1, i=1,2,3$ \\
\hline$X_{11}(a)$ & $(\mathbb{Z} / 2 \mathbb{Z})^{\oplus 2}$ & $P_{1} \cdot \Theta_{1}^{1}=P_{1} \cdot \Theta_{1}^{2}=1$ \\
& & $Q_{1} \cdot \Theta_{3}^{1}=Q_{1} \cdot \Theta_{3}^{2}=1$ \\
\hline$X_{5511}$ & $\mathbb{Z} / 5 \mathbb{Z}$ & $P_{1} \cdot \Theta_{1}^{1}=P_{1} \cdot \Theta_{2}^{2}=1$ \\
\hline$X_{4422}$ & $\mathbb{Z} / 4 \mathbb{Z} \oplus \mathbb{Z} / 2 \mathbb{Z}$ & $P_{1} \cdot \Theta_{1}^{i}=P_{1} \cdot \Theta_{0}^{4}=1, i=1,2,3$ \\
& & $Q_{1} \cdot \Theta_{2}^{1}=Q_{1} \cdot \Theta_{0}^{2}=Q_{1} \cdot \Theta_{1}^{j}=1, j=3,4$ \\
\hline$X_{3333}$ & $(\mathbb{Z} / 3 \mathbb{Z})^{\oplus 2}$ & $P_{1} \cdot \Theta_{1}^{i}=P_{1} \cdot \Theta_{0}^{4}=1, i=1,2,3$ \\
& & $Q_{1} \cdot \Theta_{1}^{i}=Q_{1} \cdot \Theta_{2}^{2}=Q_{1} \cdot \Theta_{0}^{3}=1, i=1,4$
\end{tabular}

where $K$ is a subgroup of $\operatorname{Div}(X)$ such that the class map $K \rightarrow \mathrm{Cl}(X)$ is an isomorphism (see ADHL15). Given a homogeneous element $f$ of $\mathcal{R}(X)$, we will denote its degree with $\operatorname{deg}(f) \in \mathrm{Cl}(X)$ and we will say that $f$ is the defining section of a divisor $E$ if $E=\operatorname{div}(f)+D$, where $D \in K$ is the representative for the class $\operatorname{deg}(f)$. Moreover, we will say that $\mathcal{R}(X)$ has a generator in degree $[D] \in \mathrm{Cl}(X)$ if any minimal set of generators of $\mathcal{R}(X)$ contains an element of such degree.

If $\mathcal{R}(X)$ is a finitely generated algebra, then $X$ is called a Mori dream space. By AL11, Theorem 4.2, Corollary 5.4] a rational elliptic surface $\pi: X \rightarrow \mathbb{P}^{1}$ is a Mori dream space if and only if the Mordell-Weil group of $\pi$ is finite, or equivalently if the effective cone of $X$ is rational polyhedral. We observe that if $X$ is an extremal elliptic surface, then the effective cone is generated by the classes of the negative curves of $X$, i.e., $(-1)$ - and (-2)-curves, by [AL11, Proposition 1.1].

We now recall a standard result for proving that $\mathcal{R}(X)$ is not generated in degree $[D]$, where $D$ is an effective divisor on $X$. Let $E_{1}, E_{2}$ be two disjoint curves of $X$ not linearly equivalent to $D$ and $x_{1}, x_{2} \in \mathcal{R}(X)$ be their defining sections. By Bea83, Lemma I.5] there is an exact cohomology sequence:

$$
H^{0}\left(X, D-E_{1}\right) \oplus H^{0}\left(X, D-E_{2}\right) \stackrel{g^{*}}{\rightarrow} H^{0}(X, D) \longrightarrow H^{1}\left(X, D-E_{1}-E_{2}\right),
$$

where $g^{*}(s, t)=s x_{1}+t x_{2}$.

Lemma 1.1. If $g^{*}$ is surjective, then $\mathcal{R}(X)$ has no generators in degree $[D]$. In particular, this holds if $h^{1}\left(X, D-E_{1}-E_{2}\right)=0$.

We finally state a proposition which is an immediate consequence of a result by Harbourne [Har97, Theorem III.1]. Observe that a nef divisor on a rational elliptic surface is linearly equivalent to an effective divisor by the Riemann-Roch theorem. Moreover, as a consequence of the following result, the linear system associated to a nef divisor is base point free if and only if it has no components in its base locus. 
Proposition 1.2. Let $\pi: X \rightarrow \mathbb{P}^{1}$ be a rational elliptic surface and let $D$ be a nef divisor of $X$.

(i) If $-K_{X} \cdot D>0$, then $h^{1}(X, D)=0$.

(ii) If $-K_{X} \cdot D \geq 2$, then $|D|$ is base point free.

(iii) If $-K_{X} \cdot D=1$, then $D \sim-a K_{X}+P$ for some positive integer a, where $P$ is a section of $\pi$; in this case $|D|$ contains $P$ in its base locus.

(iv) If $-K_{X} \cdot D=0$, then $D \sim-a K_{X}$ for some non-negative integer a and $h^{1}(X, D)=a$.

\section{Generators for the Cox Rings of extremal RATIONAL ELLIPTIC SURFACES}

In this section we will determine a minimal set of generators for any extremal rational elliptic surface $X$. We start proving that the degrees of the generators of $\mathcal{R}(X)$ are at the boundary of the nef cone.

Proposition 2.1. Let $\pi: X \rightarrow \mathbb{P}^{1}$ be an extremal rational elliptic surface and $D$ be an ample divisor on $X$. Then $\mathcal{R}(X)$ is not generated in degree $[D]$.

Proof. We first assume that $X$ contains two (-2)-curves $E_{1}, E_{2}$ which either belong to distinct fibers of $\pi$ and do not intersect the same section, or belong to the same fiber and have distance at least two (in the intersection graph). In this case the divisor $D-E_{1}-E_{2}$ is nef and $-K_{X} \cdot\left(D-E_{1}-E_{2}\right)=-K_{X} \cdot D>0$. Thus $h^{1}\left(D-E_{1}-E_{2}\right)=0$ by Proposition 1.2. The existence of such pair of curves can be easily verified looking at Table 1 and Table 3 for any extremal rational elliptic surface except for the surface $X_{3333}$.

If $X=X_{3333}$ let $E_{1}, E_{2}$ be two disjoint (-2)-curves and let $B:=-K_{X}+D-$ $E_{1}-E_{2}$. Since the only curve of negative self-intersection intersecting both $E_{1}$ and $E_{2}$ is a section and $D$ is ample, then $B$ is nef. Moreover, since $D-E_{1}$ is nef and $-K_{X}-E_{2} \sim E_{2}^{\prime}+E_{2}^{\prime \prime}$, where $E_{2}^{\prime}$ and $E_{2}^{\prime \prime}$ are the remaining components of the reducible fiber containing $E_{2}$, then $B^{2}=\left(D-E_{1}\right)^{2}+2\left(D-E_{1}\right) \cdot\left(E_{2}^{\prime}+E_{2}^{\prime \prime}\right)+$ $\left(E_{2}^{\prime}+E_{2}^{\prime \prime}\right)^{2} \geq 4-2>0$, so that $B$ is big. Thus $h^{1}\left(D-E_{1}-E_{2}\right)=0$ by the Kawamata-Viehweg vanishing theorem.

In both cases the statement follows from Lemma 1.1.

Remark 2.2. Observe that $X_{3333}$ is obtained blowing up the nine base points of the pencil of cubics given in Table 2, The (-2)-curves of $X$ are the proper transforms of the lines in $\mathbb{P}^{2}$ through three of the base points and the $(-1)$-curves are the exceptional divisors over such points. Thus, given two disjoint $(-2)$-curves, their images in $\mathbb{P}^{2}$ intersect at a base point of the pencil, so that both intersect the $(-1)$-curve corresponding to such point. This can be verified also computing the intersection numbers beween sections and fibers as explained in Subsection 1.1.

Definition 2.3. A connected and reduced curve $E$ of $X$ is a generalized (-1)curve if there exists a morphism to a smooth surface contracting $E$ to a point, a generalized (-2)-curve if the intersection graph on its irreducible components is a Dynkin diagram.

It follows easily from the definition and Castelnuovo's criterion Har77, Theorem $5.7]$ that a generalized $(-1)$-curve is either a $(-1)$-curve or a chain of $(-2)$-curves with a (-1)-curve at one extreme. 
Lemma 2.4. Let $N$ be a connected and reduced divisor of $X$ such that the intersection form on the irreducible components of its support is negative definite. Then $N$ is either a generalized (-2)-curve or a generalized (-1)-curve.

Proof. If $N$ only contains $(-2)$-curves, then it is a generalized (-2)-curve since its support is properly contained in a fiber of $\pi$.

Now assume that $N$ contains a $(-1)$-curve. We proceed by induction on the number $k$ of irreducible components of $N$. If $k=1$ the statement is obvious, so assume that $k>1$. Let $f: X \rightarrow Y$ be the morphism which contracts a (-1)-curve $E$ in $N$ and let $N^{\prime}=f(N)$. Since the intersection form on the support of $N$ is negative definite, then $E$ intersects exactly one other curve in the support of $N$, a (-2)-curve $R$ with $E \cdot R=1$. In fact, observe that if $E$ intersects two distinct (-2)curves $F, G$ (which are necessarily disjoint), then $(2 E+F+G)^{2}=0$. Similarly, one shows that $E$ cannot intersect any $(-1)$-curve in $N$ and that there is no $(-2)$-curve in $N$ having intersection $\geq 2$ with $E$. Thus $f(R) \subset N^{\prime}$ is a $(-1)$-curve.

Observe that $N^{\prime}$ is still a connected and reduced divisor. Moreover, the intersection form on its components is negative definite since otherwise, by pull-back, one would obtain a curve with non-negative self-intersection whose support is contained in the support of $N$. Thus, by the induction hypothesis, $N^{\prime}$ is a generalized (-1)-curve. This implies that $N$ is a generalized (-1)-curve.

2.1. Conic bundles. Let $D$ be a conic bundle, that is, an effective divisor such that $D^{2}=0,-K_{X} \cdot D=2$ and $|D|$ is base point free, so that the morphism associated to $|D|$ is a rational fibration. We now identify the conic bundles on $X$ giving generators for the Cox ring.

Proposition 2.5. Let $D$ be an effective divisor on $X$ such that $D^{2}=0$ and $|D|$ is base point free. Then either $D \sim a F, a>0$, where $F$ is a fiber of $\pi$, or $D$ is a conic bundle. Moreover, $\mathcal{R}(X)$ is generated in degree $[D]$ exactly in the cases appearing in Table 4 (where the notation is as in Table 3 ).

Proof. Since $D^{2}=0$ and $-K_{X}$ is nef, then by adjunction formula either $-K_{X} \cdot D=$ 0 and $g(D)=1$ or $-K_{X} \cdot D=2$ and $g(D)=0$. In the first case, by the Hodge index theorem, $D \sim a F$, where $F$ is a fiber of $\pi$ and $a$ is a positive integer.

Observe that $\mathcal{R}(X)$ has a generator in degree $[F]=-K_{X}$ if and only if $H^{0}\left(X,-K_{X}\right)$ is not generated by sections of negative curves, i.e., if $\pi$ has a unique reducible fiber. Similarly, if $D$ is a conic bundle, $\mathcal{R}(X)$ has a generator in degree $[D]$ if and only if the rational fibration associated to $|D|$ has a unique reducible fiber (observe that any conic bundle has at least a reducible fiber by Kleiman criterion).

Let $D$ be a reducible fiber of a conic bundle and let $D^{\text {red }}$ be the sum of its integral components. Since $-K_{X} \cdot D=2$, then $D$ either contains two $(-1)$-curves or a $(-1)$-curve with multiplicity two in its support. Let $E$ be a curve in the support of $D^{\text {red }}$ such that $D^{\text {red }}-E$ is connected. Since the intersection form on the components of $D^{r e d}-E$ is negative definite, then $D^{r e d}-E$ is either a generalized (-1)-curve or a generalized (-2)-curve by Lemma 2.4. This implies that the support of $D$ has the structure described by one of the diagrams in Figure 2 where the white vertices represent $(-1)$-curves and the remaining ones represent $(-2)$-curves. Moreover, $D$ is the unique reduced divisor with the given support and with $D^{2}=0$ : the multiplicity of each component is given by the number above each vertex in Figure 2 
TABLE 4. Generating conic bundles

\begin{tabular}{|c|c|}
\hline$X$ & $D$ \\
\hline$X_{22}, X_{211}, X_{411}, X_{9111}$ & $F$ \\
\hline$X_{22}, X_{211}$ & $2 P_{0}+2 \sum_{i=0}^{5} \Theta_{i}+\Theta_{6}+\Theta_{8}$ \\
\hline$X_{411}$ & $\begin{array}{l}P_{0}+P_{1}+\Theta_{0}+\sum_{i=2}^{6} \Theta_{i}+\Theta_{8} \\
2 P_{0}+2 \Theta_{0}+2 \sum_{i=2}^{6} \Theta_{i}+\Theta_{7}+\Theta_{8} \\
2 P_{1}+2 \sum_{i=2}^{6} \Theta_{i}+2 \Theta_{8}+\Theta_{0}+\Theta_{1}\end{array}$ \\
\hline$X_{8211}$ & $\begin{array}{l}P_{0}+P_{1}+\sum_{i \neq 1} \Theta_{i}^{1}, \\
P_{0}+P_{3}+\sum_{i \neq 7} \Theta_{i}^{1}, \\
P_{1}+P_{2}+\sum_{i \neq 3} \Theta_{i}^{1}, \\
P_{2}+P_{3}+\sum_{i \neq 5} \Theta_{i}^{1}\end{array}$ \\
\hline$X_{9111}$ & $\begin{array}{l}P_{0}+P_{2}+\sum_{i=0}^{6} \Theta_{i}, \\
P_{0}+P_{1}+\sum_{i=3}^{8} \Theta_{i}+\Theta_{0}, \\
P_{1}+P_{2}+\sum_{i=0}^{3} \Theta_{i}+\sum_{i=6}^{8} \Theta_{i}\end{array}$ \\
\hline$X_{33}, X_{321}$ & $P_{0}+P_{1}+\sum_{i=0}^{6} \Theta_{i}^{1}$ \\
\hline 1 & 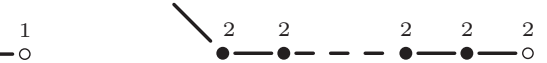 \\
\hline
\end{tabular}

FiguRE 2. Reducible fibers of conic bundles

2.2. Nef and big divisors. Let $D$ be an effective divisor without components in its base locus, $A, B$ be two smooth disjoint curves such that $D \cdot A=0$ and $s_{A}, s_{B}$ two sections defining $A$ and $B$. Then we have the following diagram:

$$
\begin{gathered}
H^{0}(X, D-B) \\
\stackrel{\downarrow}{\downarrow} \longrightarrow \stackrel{m_{B}}{\longrightarrow} H^{0}(X, D-A) \stackrel{m_{A}}{\longrightarrow} H^{0}(X, D) \stackrel{r_{A}}{\longrightarrow} H^{0}\left(A, \mathcal{O}_{A}\right) \cong \mathbb{C}
\end{gathered}
$$

where $m_{A}, m_{B}$ are the multiplication maps by $s_{A}$ and $s_{B}$ respectively, $r_{A}$ is the restriction to $A$ and the horizontal sequence is exact.

Lemma 2.6. If $h^{0}(X, D-B)>0$ and $A$ is not in the base locus of $|D-B|$, then $H^{0}(X, D)$ is generated by $H^{0}(X, D-A), H^{0}(X, D-B)$ and the defining sections of $A$ and $B$.

Proof. Observe that $D-A$ is effective and $r_{A}$ is surjective, since otherwise $|D|$ would contain components in its base locus. Since $A$ is not in the base locus of $|D-B|$, then $r_{A} \circ m_{B}$ is also surjective. Given $f \in H^{0}(X, D)$, let $r_{A}(f)=c \in H^{0}\left(A, \mathcal{O}_{A}\right)$ and let $g \in H^{0}(X, D-B)$ such that $r_{A} \circ m_{B}(g)=c$, then $f-m_{B}(g) \in H^{0}(X, D-A)$, proving the statement. 
Let $D$ be a nef and big divisor such that $|D|$ is base point free. In what follows we will denote by $D^{\perp}$ the set of negative curves orthogonal to $D$. Since $D^{2}>0$, the orthogonal complement of $D$ in $\operatorname{Pic}(X)$ is negative definite by the Hodge index theorem. Thus, if $D^{\perp}$ is not empty, the intersection form on the elements of $D^{\perp}$ is negative definite. By Lemma 2.4 each connected component of $D^{\perp}$ is either a generalized $(-1)$-curve or a generalized (-2)-curve.

Proposition 2.7. Let $D$ be a nef and big divisor such that $|D|$ is base point free and such that $D^{\perp}$ only contains $(-1)$-curves or only contains $(-2)$-curves. Then $\mathcal{R}(X)$ has no generators in degree $[D]$.

Proof. In both cases we will explain how to choose $A$ and $B$ in order to apply Lemma 2.6 .

We first assume that $D^{\perp}$ only contains $(-1)$-curves and let $A$ be a $(-1)$-curve in $D^{\perp}$. If $X$ contains another $(-1)$-curve $B$, then the divisor $D-B$ is nef. If $-K_{X} \cdot(D-B)=1$, then $D=-a K_{X}+B+B^{\prime}$ by Proposition 1.2, where $B^{\prime}$ is a $(-1)$-curve. Since $\pi$ always has a fiber with more than two components, then there exists a (-2)-curve orthogonal to both $B$ and $B^{\prime}$ (and thus to $D$ ), contradicting the hypothesis on $D$. Similarly, one can exclude the case $-K_{X} \cdot(D-B)=0$. Thus $-K_{X} \cdot(D-B) \geq 2$ and $|D-B|$ is base point free by Proposition 1.2

If $X$ only contains a $(-1)$-curve $A \in D^{\perp}$, then $X=X_{22}$ or $X_{211}$. In both cases we can choose a $(-2)$-curve $B$ which does not intersect $A$ and intersects any other $(-2)$-curve in at most one point. Observe that $D-B$ is nef and $-K_{X} \cdot(D-B)=$ $-K_{X} \cdot D \geq 2$, thus $|D-B|$ is base point free by Proposition 1.2

Finally, if $D^{\perp}$ only contains (-2)-curves, let $A$ be a (-2)-curve in $D^{\perp}$ and $B$ be a smooth fiber of $\pi$. As before, $D-B$ is nef and its linear system is base point free by Proposition 1.2

Proposition 2.8. Let $D$ be a nef and big divisor such that $|D|$ is base point free, $B$ be an integral curve in $D^{\perp}$ and $N$ be the connected component of $D^{\perp}$ which contains B. Then one of the following holds:

(i) all the components in the base locus of $|D-B|$ are contained in $N$;

(ii) $D \sim-K_{X}+B+C$, where $B$ and $C$ are distinct (-1)-curves.

Proof. Since $|D|$ is base point free, then $\varphi:=\varphi_{|D|}: X \rightarrow Y$ is a morphism onto a normal surface. Moreover, since the only negative curves of $X$ are (-1)- and (-2)curves, then $Y$ has at most ADE singularities. Let $E$ be the exceptional divisor of $\varphi$ over $\varphi(B)$. Observe that the support of $E$ is $N$ since it is the connected component of $D^{\perp}$ which contains $B$. If $E^{2}=-1$, then $E$ is a reduced divisor and $N$ is a generalized $(-1)$-curve, so that $\varphi(B)$ is a smooth point. Otherwise, $E$ is the fundamental cycle of an ADE singularity, so that $E^{2}=-2$ and $N$ is a generalized (-2)-curve (see BHPVdV04, §3, Chapter III]).

We begin by showing that $E-B$ is contained in the base locus of $|D-B|$. To this aim, consider a non-trivial decomposition $E=E_{1}+E_{2}$ where both $E_{1}$ and $E_{2}$ are effective divisors. We have that $E_{i}^{2} \leq-2$ for some $i$ since otherwise $E_{1}^{2}=E_{2}^{2}=-1$ and $E_{1} \cdot E_{2}=0$, so that $E$ would be disconnected. Hence from

$$
-2 \leq E^{2}=E_{1}^{2}+E_{2}^{2}+2 E_{1} \cdot E_{2},
$$

we deduce $E_{1} \cdot E_{2}>0$, so that some component $C$ of the support of $E_{2}$ must have negative intersection with $D-E_{1}$, which implies that $C$ is contained in the base locus of $\left|D-E_{1}\right|$. If we assume that the base locus of $|D-B|$ only contains a 
proper subdivisor $F$ of $E-B$, then we get a contradiction taking $E_{1}=B+F$. This gives the claim.

We now show that $D-E$ is nef. Let $C$ be a negative curve of $X$ with $E \cdot C>0$. First of all we observe that if $E^{2}=-1$, then $E$ is a generalized (-1)-curve and $E \cdot C^{\prime} \leq 0$ for any curve $C^{\prime}$ contained in its support. The same holds if $E^{2}=-2$ by BHPVdV04, Corollary 3.6]. Hence in both cases $C$ cannot be a component of the support $N$ of $E$. We also have $D \cdot C>0$, since otherwise $C$ would be contained in some connected component of $D^{\perp}$, disjoint from $N$, giving $E \cdot C=0$. Moreover, we have that

$$
E \cdot C \leq 2 \text {. }
$$

In case $E^{2}=-1$ this is clear since $E$ is a reduced divisor. If $E^{2}=-2$, then the support of $E$ is contained in a fiber $F$ of $\pi$. Moreover, by looking at the possible intersection graphs for $E$ (see BHPVdV04, $\S 3$, Chapter III]), we see that the multiplicity of a component of $E$ is less than or equal to the multiplicity of the same curve as a component of the fiber $F$. Thus $F-E$ is effective, so that $E \cdot C \leq 2$.

Thus we have to consider only two cases. If $E \cdot C=1$, then $(D-E) \cdot C \geq 0$ due to $D \cdot C>0$. Assume now $E \cdot C=2$. In this case $C^{2}=-2$, since $(-1)$-curves do not intersect each other and intersect a fiber of $\pi$ at one simple point. The divisor $E+C+K_{X}$ is linearly equivalent to an effective divisor by the Riemann-Roch theorem and the Serre's duality since $\left(E+C+K_{X}\right)^{2}-K_{X} \cdot\left(E+C+K_{X}\right)=0$. By the assumptions on $D$ and Proposition 1.2 we have that $D \cdot\left(-K_{X}\right) \geq 2$, thus $D \cdot(E+C) \geq 2$. Hence

$$
(D-E) \cdot C=D \cdot C-2=D \cdot(E+C)-2 \geq 0,
$$

proving that $D-E$ is nef.

We now study the base locus of $|D-E|$. We can assume $E^{2}=-1$, since otherwise $-K_{X} \cdot(D-E) \geq 2$ so that $|D-E|$ would be base point free by Proposition 1.2. Moreover, by the same proposition, the linear system $|D-E|$ is base point free unless $D-E \sim-a K_{X}+C$, where $C$ is a $(-1)$-curve of $X$, that is,

$$
D \sim-a K_{X}+E+C .
$$

In this case the unique component in the base locus of $|D-E|$ is $C$. So, either we are in case (i) of the statement or $C$ is not contained in the support $N$ of $E$ and in particular $E \cdot C \geq 0$. Assume we are in this second case. Since $E^{2}=-1$, then $D \cdot C=a+E \cdot C-1=D \cdot E=0$, so that $a=1$ and $E \cdot C=0$. Observe that $E$, which is a generalized (-1)-curve, cannot contain $(-2)$-curves, since otherwise the last $(-2)$-curve in the chain would intersect $D$ negatively contradicting the hypothesis. Thus we are in case (ii), concluding the proof.

2.3. Generators of $\mathcal{R}(X)$. We now determine a minimal generating set for the Cox ring of any extremal rational elliptic surface. We recall that $K$ is the grading group of $\mathcal{R}(X)$; see Section 1.2

Definition 2.9. Consider the following divisors:

(i) an anticanonical divisor $-K_{X}$;

(ii) smooth irreducible rational curves $C$ with $C^{2} \in\{-2,-1,0,1\}$.

For each such divisor consider the unique $D \in K$ which is linearly equivalent to it, choose a basis of $H^{0}(X, D)$ and let $\Omega$ be the union of all such bases. An element $f \in \Omega$ will be called a distinguished section of $\mathcal{R}(X)$. 
Lemma 2.10. Let $D=-K_{X}+A+B$, where $A, B$ are two distinct (-1)-curves. Then $H^{0}(X, D)$ is generated by distinguished sections.

Proof. By the Riemann-Roch theorem and Proposition 1.2 we have $h^{0}(X, D)=3$. Observe that $H^{0}(X, D)$ contains the two-dimensional subspace $f_{A} f_{B} H^{0}\left(X,-K_{X}\right)$, where $f_{A}, f_{B}$ are sections defining $A, B$ respectively. So, it is enough to show that there exists a distinguished polynomial $f$ in $H^{0}(X, D)$ such that $\operatorname{div}(f)+D$ contains neither $A$ nor $B$ in its support.

For any extremal rational elliptic surface having at least two sections $A, B$, there exists a chain of $(-2)$-curves inside one fiber of $\pi$ which connects $A$ and $B$. This can be checked looking at Table 1 and Table 3 This chain of curves, having $A, B$ as external curves, defines a conic bundle $D^{\prime}$ on $X$ (see Figure 2) such that $D-D^{\prime}$ is linearly equivalent to an effective divisor $C$ supported on negative curves. Thus we conclude taking $f=f_{C} g$, where $g$ defines a smooth element of $\left|D^{\prime}\right|$. Thus $H^{0}(X, D)$ is generated by sections of degree $\left[-K_{X}\right],\left[D^{\prime}\right]$ and by sections defining negative curves.

Theorem 2.11. Let $\pi: X \rightarrow \mathbb{P}^{1}$ be an extremal rational elliptic surface. Then the Cox ring $\mathcal{R}(X)$ is generated by distinguished sections.

Proof. Let $D$ be an effective divisor of $X$. We will prove the statement by induction on $d(D):=D \cdot H$, where $H$ is an ample divisor on $X$.

If $B$ is an integral component in the base locus of $|D|$, then $B^{2}<0$, since otherwise it would be nef and $h^{0}(X, B)>1$ by the Riemann-Roch theorem and by Proposition 1.2 (iv). Thus $B$ is either a $(-1)$-curve or a $(-2)$-curve. Moreover, the multiplication map

$$
H^{0}(X, D-B) \stackrel{\cdot f_{B}}{\longrightarrow} H^{0}(X, D)
$$

by the section $f_{B}$ defining $B$ is an isomorphism. Since $d(D-B)<d(D)$, then $H^{0}(X, D)$ is generated by distinguished sections by induction.

If $D^{2}=0$ and $|D|$ is base point free, then either $D \sim-a K_{X}$ with $a \geq 1$, or $D$ is a conic bundle by Proposition 2.5. In the first case $H^{0}(X, D)$ is generated by sections in degree $\left[-K_{X}\right]$, while in the second case it is generated by sections defining negative curves and by a section defining a smooth fiber of the conic bundle, the latter only in case $(X, D)$ belongs to the list in the statement of Proposition 2.5. In both cases $H^{0}(X, D)$ is generated by distinguished sections.

Thus in what follows we can assume $D$ to be nef, big and $-K_{X} \cdot D \geq 2$ by Proposition 1.2. Observe that we can also assume that $D$ is not ample and that $D^{\perp}$ contains both $(-1)$-curves and $(-2)$-curves by Proposition 2.1. Proposition 2.7 and the induction hypothesis.

We now assume that $D^{\perp}$ has a unique connected component $N$. Observe that $N$ is a generalized $(-1)$-curve by the previous assumptions and, since it is connected, the (-2)-curves in its support are components of a fiber $F$ of $\pi$. Let $A$ be a $(-2)$ curve in $N$.

If there exists a $(-1)$-curve $B$ such that $B \cap N=\emptyset$, then $D-B$ is nef, so that $|D-B|$ contains no (-2)-curves in the base locus by Proposition 1.2. The curves $A, B$ satisfy the hypothesis of Lemma 2.6. so that $H^{0}(X, D)$ is generated by sections in lower degrees $[D-A]$ and $[D-B]$, thus we conclude by the induction hypothesis. On the other hand, if any $(-1)$-curve intersects $N$ (this includes the 
case when all $(-1)$-curves are contained in $N)$ there are two possible cases: i) The external $(-2)$-curve of $N$ intersects a $(-1)$-curve $E$. In this case let

$$
Q=\sum_{i=1}^{r} N_{i}+E,
$$

where $N_{i}$ are the components of $N$. Observe that $Q$ is a conic bundle. We now show that $D-Q$ is nef, i.e., it has non-negative intersection with any negative curve $C$. If $C$ is contained in the support of $Q$, then $C \cdot Q=0$, so that $(D-Q) \cdot C \geq 0$. If $C$ is not contained in the support of $Q$ and $C \cdot Q \leq 1$, then clearly $(D-Q) \cdot C \geq 0$ since $D \cdot C>0$. Otherwise $C$ is not in the support of $Q, C \cdot Q=2$ and the union of $C$ with the (-2)-curves in $Q$ is the support of a fiber of $\pi$ of type $\mathrm{I}_{n}, n \geq 2$. Since $D \cdot C=D \cdot\left(-K_{X}\right) \geq 2$, again we obtain that $(D-Q) \cdot C \geq 0$. By Proposition 1.2 the curves $A, Q$ satisfy the hypothesis of Lemma 2.6. thus we conclude by the induction hypothesis.

ii) The external $(-2)$-curve of $N$ does not intersect any $(-1)$-curve. By looking at Table 3, we deduce that $X$ is isomorphic to either $X_{9111}, X_{22}$ or $X_{211}$. If $X$ is isomorphic to $X_{9111}$, since any $(-1)$-curve intersects $N$ and the external (-2)-curve of $N$ does not intersect any (-1)-curve, we can assume that

$$
N=P_{0}+\sum_{i=0}^{7} \Theta_{i} .
$$

In case $X$ is isomorphic to either $X_{22}$ or $X_{211}$, let $B$ be a smooth fiber of the conic bundle $2 P_{0}+2 \sum_{i=0}^{5} \Theta_{i}+\Theta_{6}+\Theta_{8}$. If $\Theta_{7} \notin N$, then $D-B$ is nef and Lemma 2.6 can be applied as before taking $A$ to be a (-2)-curve in $N$. Otherwise, if $\Theta_{7} \in N$, then $N=P_{0}+\sum_{i=0}^{7} \Theta_{i}$ as in the previous case.

In both cases, when the support of $N$ contains nine curves, the morphism $\varphi_{|D|}$ maps $X$ onto a smooth rational surface $S$ of Picard number one, so that $S$ is $\nu_{n}\left(\mathbb{P}^{2}\right)$, where $\nu_{n}$ is the $n$-th Veronese embedding. This implies that $D \sim n L$, where $L$ is the pull-back of a divisor generating $\operatorname{Pic}(S)$. In this case $H^{0}(X, D)=\operatorname{Sym}^{n} H^{0}(X, L)$, so that it is generated by elements in $H^{0}(X, L)$. Since a basis of $H^{0}(X, L)$ is given by distinguished sections of type (ii), then we conclude.

We now assume that $D^{\perp}$ has at least two connected components $N_{1}, N_{2}$. Let $A \in N_{1}$ and $B \in N_{2}$. By Proposition 2.8, either $A$ is not contained in the base locus of $|D-B|$ or $D \sim-K_{X}+A+B$. In the first case $H^{0}(X, D)$ is generated by $H^{0}(X, D-A), H^{0}(X, D-B)$ and by defining sections of curves with negative self-intersection by Lemma 2.6. thus we conclude by the induction hypothesis. In the second case we conclude by Lemma 2.10 .

Corollary 2.12. A minimal set of generators for $\mathcal{R}(X)$ is given by the distinguished sections which define (-1)-curves, (-2)-curves, a smooth fiber for each conic bundle having a unique reducible fiber, a smooth fiber of $\pi$ if it has a unique reducible fiber and smooth irreducible rational curves $D$ with $D^{2}=1$ such that $D^{\perp}$ contains a generalized (-1)-curve of length nine.

Proof. By Theorem 2.11 it is enough to find a set of distinguished sections which gives a minimal generating set of $\mathcal{R}(X)$. A distinguished section of degree $\left[-K_{X}\right]$ is contained in such a set if and only if the elliptic fibration has a unique reducible fiber. Similarly, the only conic bundles giving generators of $\mathcal{R}(X)$ are those with a unique reducible fiber, which have been classified in Proposition 2.5 Finally, if $D$ 
is a smooth irreducible rational curves $D$ with $D^{2}=1$, then $\varphi:=\varphi_{|D|}: X \rightarrow \mathbb{P}^{2}$ is a birational morphism. In this case $H^{0}(X, D)=\varphi^{*} H^{0}\left(\mathbb{P}^{2}, \mathcal{O}_{\mathbb{P}^{2}}(1)\right)$ is generated by sections defining negative curves unless the exceptional divisor of $\varphi$ contracts a generalized (-1)-curve of length nine to one point. A divisor $D$ with this property only exists for the surfaces $X_{22}, X_{211}$ and $X_{9111}$ (in the last case there are three such divisors, exchanged by the Mordell-Weil group).

\section{Elliptic surfaces of COMPlexity one}

This section deals with extremal elliptic surfaces $X$ which admit an action of the torus $T=\mathbb{C}^{*}$. These are the surfaces $X_{22}, X_{33}, X_{44}$ and the surfaces $X_{11}(a)$, where $a \in \mathbb{C}-\{0,1\}$ (see Dui10, Proposition 9.2.17]). The action of the torus is given as follows, where $c \in T$ :

$$
\begin{array}{lll}
X_{22}: & {\left[x_{0}: x_{1}: x_{2}\right] \mapsto\left[c x_{0}: x_{1}: c^{-2} x_{2}\right],} \\
X_{33}: & {\left[x_{0}: x_{1}: x_{2}\right] \mapsto\left[c x_{0}: x_{1}: c^{-1} x_{2}\right],} \\
X_{44}: & {\left[x_{0}: x_{1}: x_{2}\right] \mapsto\left[c x_{0}: x_{1}: x_{2}\right],} \\
X_{11}(a): & {\left[x_{0}: x_{1}: x_{2}\right] \mapsto\left[c x_{0}: x_{1}: x_{2}\right] .}
\end{array}
$$

We will compute the Cox ring of such surfaces by means of Hau13, Theorem 3.18]. We recall that a fixed point for the T-action is called elliptic if it lies in the closure of infinitely many orbits. In order to compute the Cox ring we proceed in two steps, following the technique explained in [Hau13, §3.3]: first of all we produce a $T$-equivariant blow-up $\phi: X^{\prime} \rightarrow X$ such that $X^{\prime}$ does not contain any elliptic fixed point, then we construct the intersection graph of the negative curves of $X^{\prime}$, i.e., the Orlik-Wagreich graph of $X^{\prime}$. This information is enough to give a presentation of the Cox ring of $X^{\prime}$, from which one obtains a presentation for the Cox ring of $X$ putting equal to 1 the variables corresponding to the exceptional divisors of $\phi$. We will discuss in detail one example, the other ones being similar.

3.1. The surface $X_{22}$. The surface $X_{22}$ contains one elliptic fixed point at the cusp of the fiber of type II, whose plane model is the curve of equation $x_{1}^{3}+x_{2} x_{0}^{2}=0$, and a curve of fixed points which corresponds to the trivalent component of the fiber of type II* $^{*}$ Blowing-up the cusp three times we obtain a surface $X_{22}^{\prime}$ which does not contain elliptic points. The intersection graph of its negative curves is given in Figure 3. The four right-hand side vertices come from the blow-up of the cusp (the $(-1)$-curve there is pointwise fixed), the left-hand side is the graph of the fiber of type $\mathrm{II}^{*}$, while the central $(-1)$-curves are orbits whose self-intersections are uniquely determined by the following conditions on the Hirzebruch-Jung continued fractions:

$$
[2,2,2,2,2,1,6]=0,[2,1,2]=0,[2,2,1,3]=0 .
$$

The partial quotients of the previous continued fractions give the orders of the isotropy subgroups of the $T$-action on the generic points of the negative curves of $X_{22}^{\prime}$ according to Hau13, pg. 31]. This allows us to write down a presentation for $\mathcal{R}\left(X_{22}^{\prime}\right)$ by [Hau13, Theorem 3.18] and of $\mathcal{R}\left(X_{22}\right)$ by putting equal to 1 the variables corresponding to the exceptional divisors of the blow-up $X_{22}^{\prime} \rightarrow X_{22}$ (those corresponding to vertices with a white circle in Figure 3). The ring $\mathcal{R}\left(X_{22}\right)$ is given in Table 5 where the variable $T_{7}$ is the defining section of the left trivalent vertex in the graph and $T_{8}, \ldots, T_{13}, T_{4}, T_{1}, T_{3}, T_{6}, T_{5}, T_{2}$ are the defining sections of the remaining black vertices, ordered row by row from the left to the right. 


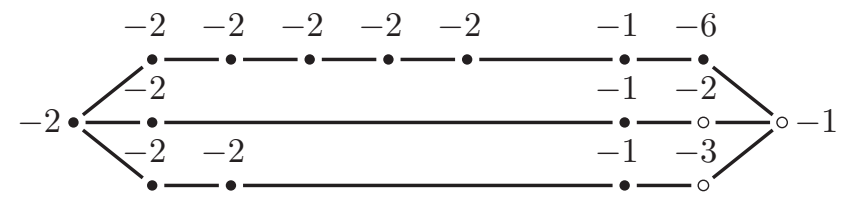

Figure 3 . The surface $X_{22}$

We observe that the sections $x_{1}, x_{0}, x_{2}, x_{1}^{3}+x_{0}^{2} x_{2}$ define the images in $\mathbb{P}^{2}$ of the curves defined by $T_{2}, T_{3}, T_{1}$ and $T_{4}$ respectively. The unique relation of the Cox ring corresponds to the relation among the three cubics $x_{1}^{3}, x_{0}^{2} x_{2}, x_{1}^{3}+x_{0}^{2} x_{2}$, which are elements of the pencil of cubics having $x_{2}=0$ as an inflectional tangent in $(1: 0: 0)$ and which have a cusp at $(0: 0: 1)$ whose principal tangent is $x_{0}=0$.

3.2. The surface $X_{33}$. The surface $X_{33}$ contains one elliptic fixed point, the singular point of the fiber of type III, and a curve of fixed points, given by the trivalent component of the fiber of type $\mathrm{III}^{*}$. Blowing-up the elliptic point twice we obtain the surface $X^{\prime}$ whose intersection graph of negative curves is given in Figure 4. The ring $\mathcal{R}\left(X_{33}\right)$ is thus computed as before and is given in Table 5 where the variable $T_{6}$ is the defining section of the left trivalent vertex in the graph and $T_{1}, T_{11}, T_{12}, T_{13}, T_{2}, T_{5}, T_{4}, T_{7}, T_{8}, T_{9}, T_{10}, T_{3}$ are the defining sections of the remaining black vertices, ordered row by row from the left to the right.

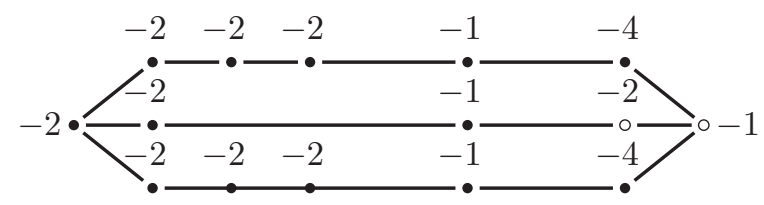

Figure 4 . The surface $X_{33}$

3.3. The surface $X_{44}$. The surface $X_{44}$ contains one elliptic fixed point which is the singular point of the fiber of type IV. Blowing-up this point once we obtain the surface $X_{44}^{\prime}$ whose graph of negative curves is given in Figure 5 The ring $\mathcal{R}\left(X_{44}\right)$ is thus computed as before and is given in Table 5 where the variable $T_{1}$ is the defining section of the left trivalent vertex in the graph and $T_{5}, T_{6}, T_{7}, T_{2}, T_{8}, T_{9}, T_{10}, T_{3}, T_{11}, T_{12}$, $T_{13}, T_{4}$ are the defining sections of the remaining black vertices, ordered row by row from the left to the right.

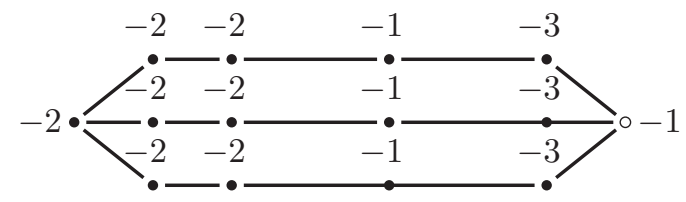

Figure 5. The surface $X_{44}$ 
3.4. The surface $X_{11}(a)$. In this case we have a one parameter family of surfaces $X_{11}(a), a \in \mathbb{C}-\{0,1\}$. The surface $X_{11}(a)$ does not contain elliptic fixed points and its graph of negative curves is given in Figure 6 where the variables $T_{12}$ and $T_{4}$ are the defining sections of the left fourvalent vertex and of the right fourvalent vertex respectively and $T_{1}, T_{7}, T_{6}, T_{2}, T_{9}, T_{8}, T_{3}, T_{11}, T_{10}, T_{13}, T_{14}, T_{5}$ are the defining sections of the remaining vertices, ordered row by row from the left to the right. In this case the Cox ring, which is given in Table 5, has two relations in the same degree. These relations correspond to the relations among the four lines $x_{1}=0$, $x_{2}=0, x_{1}-x_{2}=0, x_{1}-a x_{2}=0$ of the pencil of lines in $\mathbb{P}^{2}$ through $(1: 0: 0)$.

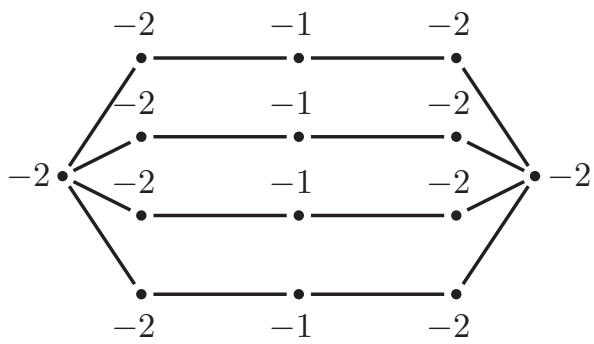

FiguRE 6 . The surface $X_{11}(a)$

\section{Relations of the Cox Ring}

In this section we will determine the ideal of relations $I(X)$ of $\mathcal{R}(X)$, where $X$ is an extremal rational elliptic surface, following [BHKS, Construction 3.1].

4.1. Algebraic preliminaries. Let $\mathbb{C}[T, S]$ be a polynomial ring in $m+n$ variables $T_{1}, \ldots, T_{m}, S_{1}, \ldots, S_{n}$ graded by an abelian group $K_{T} \oplus K_{S}$, where $K_{S}$ is free. We assume that the degrees of the $S$ variables give a basis of $K_{S}$. In what follows $\mathbb{C}[T]$ denotes the polynomial ring in the first $m$ variables graded by $K_{T}, \mathbb{C}\left[T, S^{ \pm 1}\right]$ is the localization of $\mathbb{C}[T, S]$ with respect to all the $S$ variables and $\mathbb{C}\left[T, S^{ \pm 1}\right]_{0}$ is its degree zero part with respect to the $K_{S}$ grading. Assume the following diagram of homomorphisms is given:

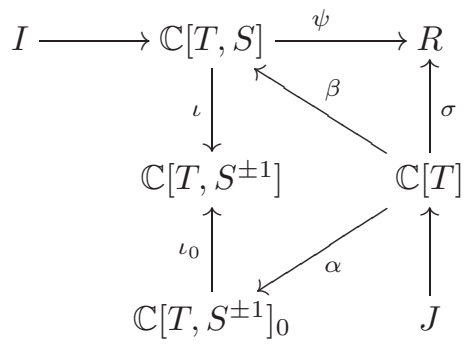

where $\psi$ is a graded surjective homomorphism to a $K_{T} \oplus K_{S}$-graded domain $R$ with kernel $I, \sigma$ is a $K_{T}$-graded homomorphism with kernel $J, \iota$ and $\iota_{0}$ are the natural inclusions, $\sigma=\psi \circ \beta$ and

$$
\iota_{0} \circ \alpha\left(T_{i}\right)=\iota \circ \beta\left(T_{i}\right)=T_{i} \cdot m_{i}(S),
$$


where $m_{i}(S) \in \mathbb{C}[S]$ is the unique monomial in the variables $S$ such that $T_{i} \cdot m_{i}(S)$ has $K_{S}$ degree equal to zero (the uniqueness follows from the hypothesis on the degrees of the $S$ variables). We observe that $\alpha$ is an isomorphism.

Proposition 4.1. Let $J^{\prime} \subset \mathbb{C}[T, S]$ be the extension and contraction of the ideal $\alpha(J)$ (by $\iota_{0}$ and $\left.\iota\right)$. Then $J^{\prime} \subset I$.

Proof. Observe that $\beta(J) \subset I$ since $\sigma=\psi \circ \beta$. Moreover, from the equality

$$
\beta(J) \cdot \mathbb{C}\left[T, S^{ \pm 1}\right]=\alpha(J) \cdot \mathbb{C}\left[T, S^{ \pm 1}\right]
$$

we get that $J^{\prime}$ is contained in the saturation of $I$ with respect to the variables $S$. Since $R$ is a domain, then $I$ is saturated, hence we get the statement.

4.2. Cox rings of blow-ups. Let $f: X \rightarrow Y$ be a blow-up morphism, where $X$ and $Y$ are smooth Mori dream spaces. Let $T_{1}, \ldots, T_{m}, S_{1}, \ldots, S_{n}$ be a minimal set of generators of $\mathcal{R}(X)$, where $S_{1}, \ldots, S_{n}$ define the irreducible components of the exceptional divisor of $f$. The polynomial ring $\mathbb{C}[T, S]$ is graded by $\mathrm{Cl}(X)=$ $K_{T} \oplus K_{S}$, where $K_{T}=f^{*} \mathrm{Cl}(Y)$ and $K_{S}$ is the free abelian group generated by the degrees of the $S$ variables.

In this setting we take $R=\mathcal{R}(X)$ and $\psi: \mathbb{C}[T, S] \rightarrow \mathcal{R}(X)$ to be the natural quotient homomorphism with kernel $I(X)$. Observe that, since $\beta\left(T_{i}\right)$ has degree zero with respect to the $K_{S}$-grading, then $\psi \circ \beta\left(T_{i}\right)=f^{*}\left(s_{i}\right)$ via the pull-back homomorphism $f^{*}: \mathcal{R}(Y) \rightarrow \mathcal{R}(X)$. Thus we obtain the following result.

Corollary 4.2. Let $J$ be the kernel of the $K_{T}$-graded homomorphism

$$
\phi: \mathbb{C}[T] \rightarrow \mathcal{R}(Y), \quad T_{i} \mapsto s_{i} .
$$

Then the ideal $J^{\prime}$ defined before is contained in $I(X)$ and $J^{\prime}=I(X)$ if $\operatorname{dim} J^{\prime}=$ $\operatorname{dim} I(X)$.

Proof. By definition $\sigma=f^{*} \circ \phi$. Since $f^{*}$ is injective, the first claim in the statement follows from Proposition 4.1. By [BHKS, Proposition 3.3] $J^{\prime}$ is prime since $J$ is prime. Thus the second claim follows from the fact that $I(X)$ is prime.

We recall that the quotient of an open subset of the affine variety $\operatorname{Spec} \mathcal{R}(X)$ for the action of a torus of dimension $\operatorname{rank} \mathrm{Cl}(X)$ is isomorphic to $X$ [ADHL15], thus $\operatorname{dim} I(X)=\operatorname{rank} \mathrm{Cl}(X)+2$.

4.3. The ideal of relations of rational elliptic surfaces. We recall that any rational elliptic surface $X$ is a blow-up of the projective plane at nine points, eventually infinitely near. Fix a blow-up morphism $X \rightarrow \mathbb{P}^{2}$, a natural basis for the Picard group of $X$ is given by $e_{0}, e_{1}, \ldots, e_{9}$, where $e_{0}$ is the pull-back of the hyperplane class of $\mathbb{P}^{2}$ and the $e_{i}$ 's with $i \geq 1$ are the classes of the exceptional divisors with $e_{i} \cdot e_{j}=-\delta_{i j}$ (observe that the exceptional divisors with classes $e_{1}, \ldots, e_{9}$ are not necessarily irreducible if there are infinitely near points). Thus in this case $K_{T} \cong \mathbb{Z}$ and $K_{S} \cong \mathbb{Z}^{9}$.

Example 4.3. We will apply Corollary 4.2 to compute the ideal of relations of the Cox ring of the surface $X=X_{411}$. We recall that $X$ has an elliptic fibration with a unique reducible fiber of type $I_{4}^{*}$ and Mordell-Weil group of order two. The surface can be obtained from the following pencil of cubic curves:

$$
\left(x_{0}^{2} x_{1}+x_{2}^{3}+x_{1}^{2} x_{2}\right)+t x_{1} x_{2}^{2}=0 .
$$


Let $l_{1}: x_{1}=0, l_{2}: x_{2}=0$ and $p=(1: 0: 0)$ be their intersection point. We call $C$ the smooth cubic given by $t=0$. Observe that the line $l_{2}$ is tangent to $C$ in $q=(0: 1: 0)$ and the line $l_{1}$ is the inflectional tangent of $C$ at $p$. The surface $X$ is obtained blowing-up $\mathbb{P}^{2}$ five times at $p$ (that is, at $p$ and at four points infinitely near to it) and four times at $q$. We denote by $e_{1}, \ldots, e_{5}$ the classes of the exceptional divisors over $p$ and by $e_{6}, \ldots, e_{9}$ those over $q$, where $e_{i} \cdot e_{j}=-\delta_{i j}$ and all the $e_{i}$ 's are reducible except for $e_{5}$ and $e_{9}$, which are taken to be the exceptional divisors of the last blow-up over $p$ and $q$. By Theorem 2.11 a set of generators for the Cox ring of $X$ is given by

- the sections $T_{1}, T_{2}$ defining the proper transforms of the lines $l_{1}$ and $l_{2}$, whose degrees are:

$$
\left[\Theta_{1}\right]=e_{0}-e_{1}-e_{2}-e_{3}, \quad\left[\Theta_{5}\right]=e_{0}-e_{1}-e_{6}-e_{7}
$$

- three sections $T_{3}, T_{4}, T_{5}$ defining smooth fibers of the conic bundles whose degrees are:

$$
\begin{aligned}
& {\left[\Theta_{0}+\Theta_{8}+\sum_{i=2}^{6} \Theta_{i}+P_{0}+P_{1}\right]=e_{0}-e_{6},} \\
& {\left[2 P_{0}+2 \Theta_{0}+2 \sum_{i=2}^{6} \Theta_{i}+\Theta_{7}+\Theta_{8}\right]=2 e_{0}-e_{6}-e_{7}-e_{8}-e_{9},} \\
& {\left[2 P_{1}+2 \Theta_{8}+2 \sum_{i=2}^{6} \Theta_{i}+\Theta_{0}+\Theta_{1}\right]=3 e_{0}-\left(e_{1}+\cdots+e_{5}\right)-2 e_{6} ;}
\end{aligned}
$$

- the section $T_{6}$ defining the proper transform of the cubic $C$, of degree $3 e_{0}-$ $\sum_{i=1}^{9} e_{i}$

- the sections $T_{7}, \ldots, T_{15}$ defining the irreducible components of the exceptional divisors over $p$ and $q$, whose degrees are:

$$
\begin{array}{llll}
{\left[\Theta_{0}\right]=e_{4}-e_{5},} & {\left[\Theta_{i}\right]=e_{5-i}-e_{6-i},} & {\left[P_{0}\right]=e_{5}} & (i=2,3,4), \\
{\left[\Theta_{6}\right]=e_{7}-e_{8},} & {\left[\Theta_{7}\right]=e_{6}-e_{7},} & {\left[\Theta_{8}\right]=e_{8}-e_{9},} & {\left[P_{1}\right]=e_{9} .}
\end{array}
$$

As a smooth fiber of the first conic bundle we can choose the proper transform of the line $x_{0}=0$. Moreover, the proper transform of the conic $x_{0}^{2}+x_{1} x_{2}=0$ is smooth of degree $2 e_{0}-e_{6}-e_{7}-e_{8}-e_{9}$ and the proper transform of the cubic $x_{0}^{2} x_{1}+x_{2}^{3}=0$ is smooth of degree $3 e_{0}-\left(e_{1}+\cdots+e_{5}\right)-2 e_{6}$, thus their defining sections can be taken to be generators of $\mathcal{R}(X)$. With these choices, the sections $s_{1}, \ldots, s_{6}$ defining the image of the curves defined by $T_{1}, \ldots, T_{6}$ are the following:

$$
\begin{gathered}
s_{1}=x_{1}, s_{2}=x_{2}, s_{3}=x_{0}, s_{4}=x_{0}^{2}+x_{1} x_{2}, \\
s_{5}=x_{0}^{2} x_{1}+x_{2}^{3}, s_{6}=x_{0}^{2} x_{1}+x_{2}^{3}+x_{1}^{2} x_{2} .
\end{gathered}
$$

A computation with Magma [BCP97] shows that a set of generators for the ideal $J$ is:

$$
T_{1} T_{2}+T_{3}^{2}-T_{4}, T_{1} T_{4}+T_{2}^{3}-T_{6}, T_{1} T_{3}^{2}+T_{2}^{3}-T_{5},
$$

and gives a set of generators for the ideal $J^{\prime}$. This set is the one given in Table 5 in fact, $J^{\prime}=I(X)$ since a computation with the same program shows that $\operatorname{dim} J^{\prime}=12=\operatorname{dim} I(X)$.

Remark 4.4. The set of generators of the ideal $J$ given above is induced by geometric relations among curves in $\mathbb{P}^{2}$. In fact, the three conics $s_{1} s_{2}, s_{3}^{2}$ and $s_{4}$ belong to the pencil of conics whose tangent in $(0: 1: 0)$ is $x_{2}=0$ and whose tangent in $(0: 0: 1)$ is $x_{1}=0$, thus there exists a linear relation among them, which indeed corresponds to the first generator of $J$. The three cubics $s_{1} s_{4}, s_{2}^{3}$ and $s_{6}$ belong to the pencil of cubics which pass through $(1: 0: 0)(\operatorname{resp}(0: 0: 1))$ and are osculating to the same cubic, $x_{0}^{2} x_{1}+x_{1}^{2} x_{2}+x_{2}^{3}=0$, with multiplicity 3 (resp. 6) 
in this point. Thus there exists a linear relation among them, which corresponds to the second generator of $J$. Finally, the three cubics $s_{1} s_{3}^{2}, s_{2}^{3}$ and $s_{5}$ belong to the pencil of cubics with a cusp in $(0: 1: 0)$ whose principal tangent is $x_{0}=0$ and which are osculating with multiplicity 3 to the cubic $x_{0}^{2} x_{1}+x_{2}^{2}$ in $(1: 0: 0)$. The linear relation between them corresponds to the third generator of $J$.

The procedure in the previous example allows us to compute the ideal of relations of the Cox ring of all the extremal rational elliptic surfaces, except for the surfaces $X_{8211}, X_{9111}$ and $X_{4422}$, where we are not able to identify the ideal $J^{\prime}$ for computational reasons. The Magma code for these computations is available here

$$
\text { http://www2.udec.cl/alaface/software/coxrs.html }
$$

Theorem 4.5. The Cox rings of all extremal rational elliptic surfaces are given in Table [5.

Proof. In case the surface has complexity one, its Cox ring is computed in Section 3. Otherwise, the Cox rings are computed by means of Theorem 2.11 and Corollary 4.2. In order to apply Corollary 4.2, we recall that here $Y=\mathbb{P}^{2}$ and the birational morphism $f: X \rightarrow Y$ is chosen to be the blow-up of the base locus of one of the pencils of cubics given in Table 2. The sections $s_{1}, \ldots, s_{m} \in \mathbb{C}\left[x_{0}, x_{1}, x_{2}\right]$ defining the homomorphism $\phi$ in Corollary 4.2 are the following:

$$
\begin{array}{l|l}
X_{411} & x_{1}, x_{2}, x_{0}, x_{0}^{2}+x_{1} x_{2}, x_{0}^{2} x_{1}+x_{2}^{3}, x_{0}^{2} x_{1}+x_{2}^{3}+x_{1}^{2} x_{2} \\
X_{222} & x_{0}-x_{1}, x_{0}, x_{1}, x_{1}-x_{2}, x_{1}^{3}+2 x_{0} x_{1} x_{2}-2 x_{1}^{2} x_{2}-x_{0} x_{2}^{2}+x_{1} x_{2}^{2}, \\
& x_{1}^{3}+2 x_{0} x_{1} x_{2}-2 x_{1}^{2} x_{2}-x_{0} x_{2}^{2}+x_{1} x_{2}^{2}+x_{0} x_{1}\left(x_{0}-x_{1}\right) \\
X_{141} & x_{0}, x_{1}, x_{0}-x_{1}, x_{2}, x_{0} x_{1}-x_{1}^{2}+x_{0} x_{2} \\
X_{6321} & x_{0}, x_{1}, x_{2}, x_{0}+x_{2}, x_{0}+x_{1}, x_{1}+x_{2}, x_{0}+x_{1}+x_{2}, x_{0} x_{1}+x_{0} x_{2}+x_{1} x_{2} \\
X_{5511} & x_{0}, x_{2}, x_{1}, x_{0}+x_{1}, x_{0}+x_{1}+x_{2}, x_{1}+x_{2} \\
X_{3333} & x_{2}, x_{1}, x_{0}, x_{0}+x_{1}+x_{2}, x_{0}+x_{1}+\epsilon^{2} x_{2}, \\
& x_{0}+x_{1}+\epsilon x_{2}, x_{0}+\epsilon^{2} x_{1}+x_{2}, x_{0}+\epsilon^{2} x_{1}+\epsilon^{2} x_{2}, \\
& x_{0}+\epsilon^{2} x_{1}+\epsilon x_{2}, x_{0}+\epsilon x_{1}+x_{2}, x_{0}+\epsilon x_{1}+\epsilon^{2} x_{2}, x_{0}+\epsilon x_{1}+\epsilon x_{2}
\end{array}
$$

where $\epsilon$ is a primitive cube root of unity.

Remark 4.6. The ideal of relation of the surface $X_{3333}$ is given up to the action of its automorphism group $G$, the Hessian group [AD09].

\section{Applications}

Let $X$ be an extremal rational elliptic surface. Any contraction of $(-1)$-curves and (-2)-curves gives a birational morphism $\varphi: X \rightarrow Y$, where $Y$ is still a Mori dream space by [Ka, Theorem 1.1]. The morphism $\varphi$ induces an isomorphism $\varphi_{*}: \mathbb{C}(X) \rightarrow \mathbb{C}(Y)$ between the fields of rational functions. This allows us to define a homomorphism

$$
\varphi_{*}: \mathcal{R}(X) \rightarrow \mathcal{R}(Y), \quad f \mapsto \varphi_{*} f,
$$

where $\operatorname{deg}\left(\varphi_{*} f\right):=\varphi_{*}(\operatorname{deg}(f))$. Moreover, in case the pull-back of divisors is well defined, for example if $Y$ is smooth, one can consider the pull-back homomorphism $\varphi^{*}: \mathcal{R}(Y) \rightarrow \mathcal{R}(X)$. Since $\varphi_{*} \circ \varphi^{*}=\mathrm{id}_{\mathcal{R}(Y)}$, then $\varphi_{*}$ is surjective.

Let $f \in \mathcal{R}(X)$ be an element defining an integral component $E$ of the exceptional divisor of $\varphi$. If $E \in K$, as we can always assume in our setting, then $f$ can be taken 
to be a constant equal to 1 . The kernel of $\varphi_{*}$ clearly contains the ideal $I$ generated by the elements $f-1$, where $f$ is as before in degree $E$ and 1 is in degree 0 . Thus $\varphi_{*}$ factorizes through a surjective homomorphism $\mathcal{R}^{\prime} \rightarrow \mathcal{R}(Y)$, where $\mathcal{R}^{\prime}=\mathcal{R}(X) / I$. It follows that $\mathcal{R}^{\prime} \cong \mathcal{R}(Y)$ if $\operatorname{dim} \mathcal{R}^{\prime}=\operatorname{dim} \mathcal{R}(Y)$ and $\operatorname{Spec}\left(\mathcal{R}^{\prime}\right)$ is irreducible. The latter properties can be checked by means of a computer program; for example, with SINGULAR DGPS15.

This criterion allows us to compute the Cox ring of several weak del Pezzo surfaces which can be obtained contracting $(-1)$-curves of extremal rational elliptic surfaces. We will give only two examples here.

Example 5.1. The Cayley's cubic surface $Y$ is the only cubic surface in $\mathbb{P}^{3}$ with four ordinary double points:

$$
Y: \frac{1}{x_{0}}+\frac{1}{x_{1}}+\frac{1}{x_{2}}+\frac{1}{x_{3}}=0 .
$$

The surface is constructed as follows. Let $l_{1}, l_{2}, l_{3}, l_{4}$ be the defining polynomials of four general lines of $\mathbb{P}^{2}$ whose unique linear relation is $l_{1}+l_{2}+l_{3}+l_{4}=0$. Then the Cayley cubic $Y$ is the image of the rational map $\mathbb{P}^{2} \rightarrow \mathbb{P}^{3}$ defined by

$$
\left(x_{0}: x_{1}: x_{2}\right) \mapsto\left(l_{1} l_{2} l_{3}: l_{1} l_{2} l_{4}: l_{1} l_{3} l_{4}: l_{2} l_{3} l_{4}\right) .
$$

Indeed, the products of three of the above reducible cubics equal $l_{i}\left(l_{1} l_{2} l_{3} l_{4}\right)^{2}$, for $i \in\{1,2,3,4\}$, and thus their sum is zero by construction. Hence the minimal resolution $\tilde{Y}$ of $Y$ is the blow-up of $\mathbb{P}^{2}$ along the six points of intersection of the four lines. In particular, $\tilde{Y}$ contains four $(-2)$-curves, corresponding to the four lines, and nine $(-1)$-curves corresponding to the six intersection points plus the strict transforms of three lines through pairs of these points.

Contracting the three $(-1)$-curves of $X_{6321}$ corresponding to the generators $T_{10}$, $T_{12}, T_{14}$, with the notation in Table 5 , it is easy to verify that one obtains a smooth surface whose configuration of negative curves is exactly the one of $\tilde{Y}$. Hence the Cox ring of $\tilde{Y}$ is obtained by that of $X_{6321}$ by putting equal to one the variables $T_{10}, T_{12}, T_{14}$. In this case $\mathcal{R}(\tilde{Y})$ has 13 generators, which correspond to the negative curves, and the ideal of relations is

$$
I(\tilde{Y})=\left\langle\begin{array}{c}
T_{1} T_{11} T_{15}+T_{3} T_{13} T_{17}-T_{4} T_{16}, T_{1} T_{9} T_{15}+T_{2} T_{13} T_{16}-T_{5} T_{17}, \\
T_{2} T_{11} T_{16}+T_{3} T_{9} T_{17}-T_{6} T_{15}, T_{1} T_{9} T_{11}+T_{6} T_{13}-T_{7} T_{16} T_{17}, \\
T_{3} T_{9} T_{13}+T_{5} T_{11}-T_{7} T_{15} T_{16}, T_{2} T_{11} T_{13}+T_{4} T_{9}-T_{7} T_{15} T_{17}, \\
T_{1} T_{3} T_{9}^{2}+T_{2} T_{7} T_{16}^{2}-T_{5} T_{6}, T_{1} T_{2} T_{11}^{2}+T_{3} T_{7} T_{17}^{2}-T_{4} T_{6}, \\
T_{1} T_{7} T_{15}^{2}+T_{2} T_{3} T_{13}^{2}-T_{4} T_{5}
\end{array}\right\rangle .
$$

This gives an alternative proof of the result given in [HB03, §2]. The relations above are induced by the pencils of lines through $p_{1}, \ldots, p_{6}$ and by the pencils of conics through four of the points (such that no three of them are collinear). In fact each such pencil clearly contains three sections which are polynomials in the generators of the Cox ring.

Example 5.2. In Der14 Derenthal classified the weak del Pezzo surfaces whose Cox ring has only one relation and described their Cox rings. An easy check shows that, by contracting $(-1)$-curves on extremal rational elliptic surfaces, a surface for each of the types in [Der14, Theorem 2] can be obtained. This allows us to give an alternative computation of their Cox ring in several cases. For example, it is known that there are only two del Pezzo surfaces $Y_{0}, Y_{1}$ of degree one and type $E_{8}$ up to isomorphism [AN06]. These can be obtained by contracting the unique section in 
the surface $X_{22}$ and in the surface $X_{211}$ respectively. Their Cox rings are generated by $T_{1}, \ldots, T_{12}$ and the ideal of relations is:

$$
I\left(Y_{\lambda}\right)=\left\langle T_{1} T_{3}^{2}+T_{2}^{3} T_{5}^{2} T_{6}+T_{4} T_{8} T_{9}^{2} T_{10}^{3} T_{11}^{4} T_{12}^{5}+\lambda T_{1} T_{2}^{2} T_{5}^{2} T_{6}^{2} T_{7}^{2} T_{8}^{2} T_{9}^{2} T_{10}^{2} T_{11}^{2} T_{12}^{2}\right\rangle,
$$

with $\lambda=0,1$.

Remark 5.3. In Der14] and [HB03 the explicit description of the Cox ring of certain weak del Pezzo surfaces has been used to study the Manin's conjecture on the asymptotic behavior of the number of rational points of bounded height on such surfaces, following a method introduced by Salberger Sal98.

\section{The Cox RIngs}

TABLE 5. The Cox rings of extremal rational elliptic surfaces

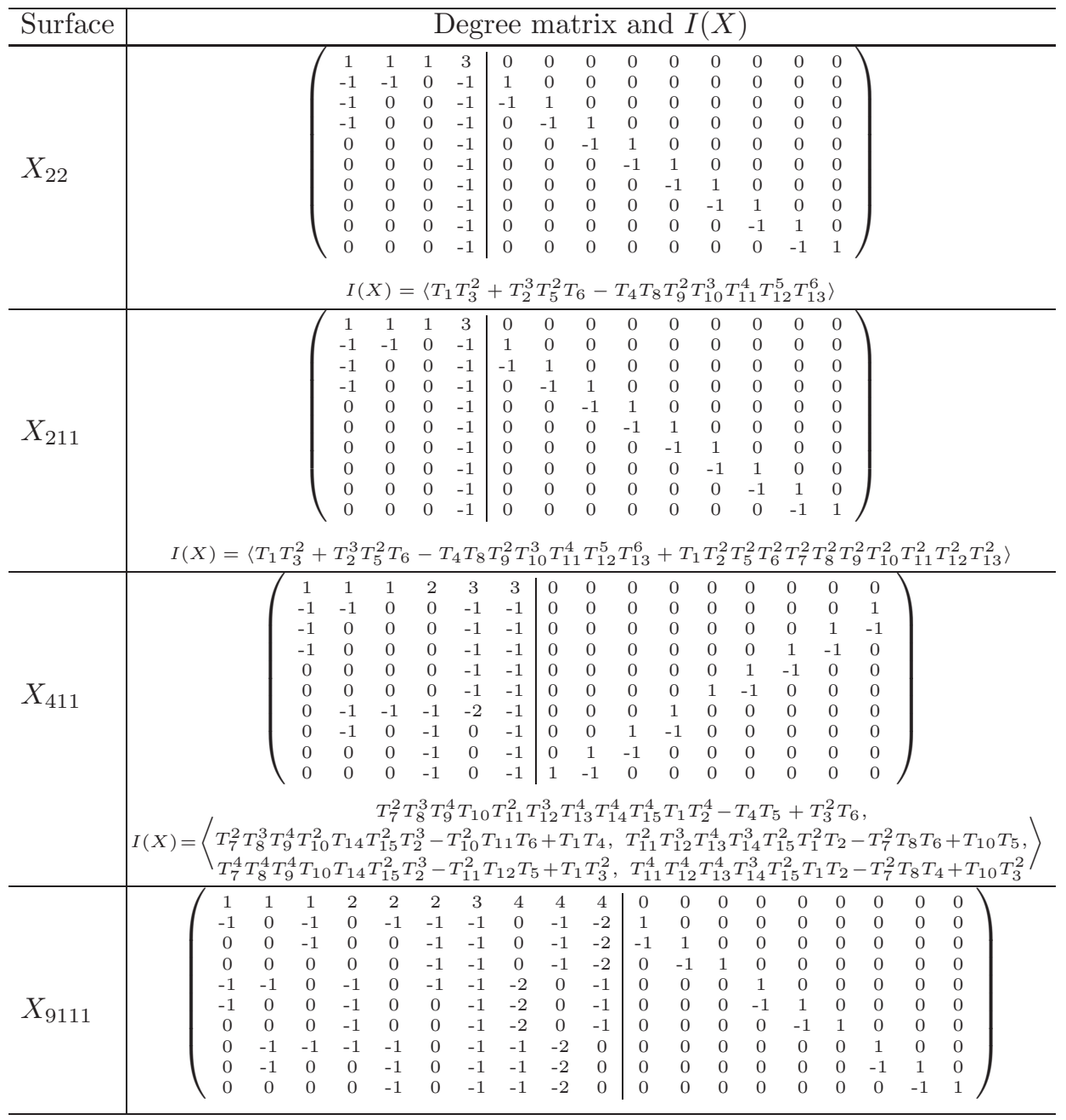


TABLE 5. (continued)

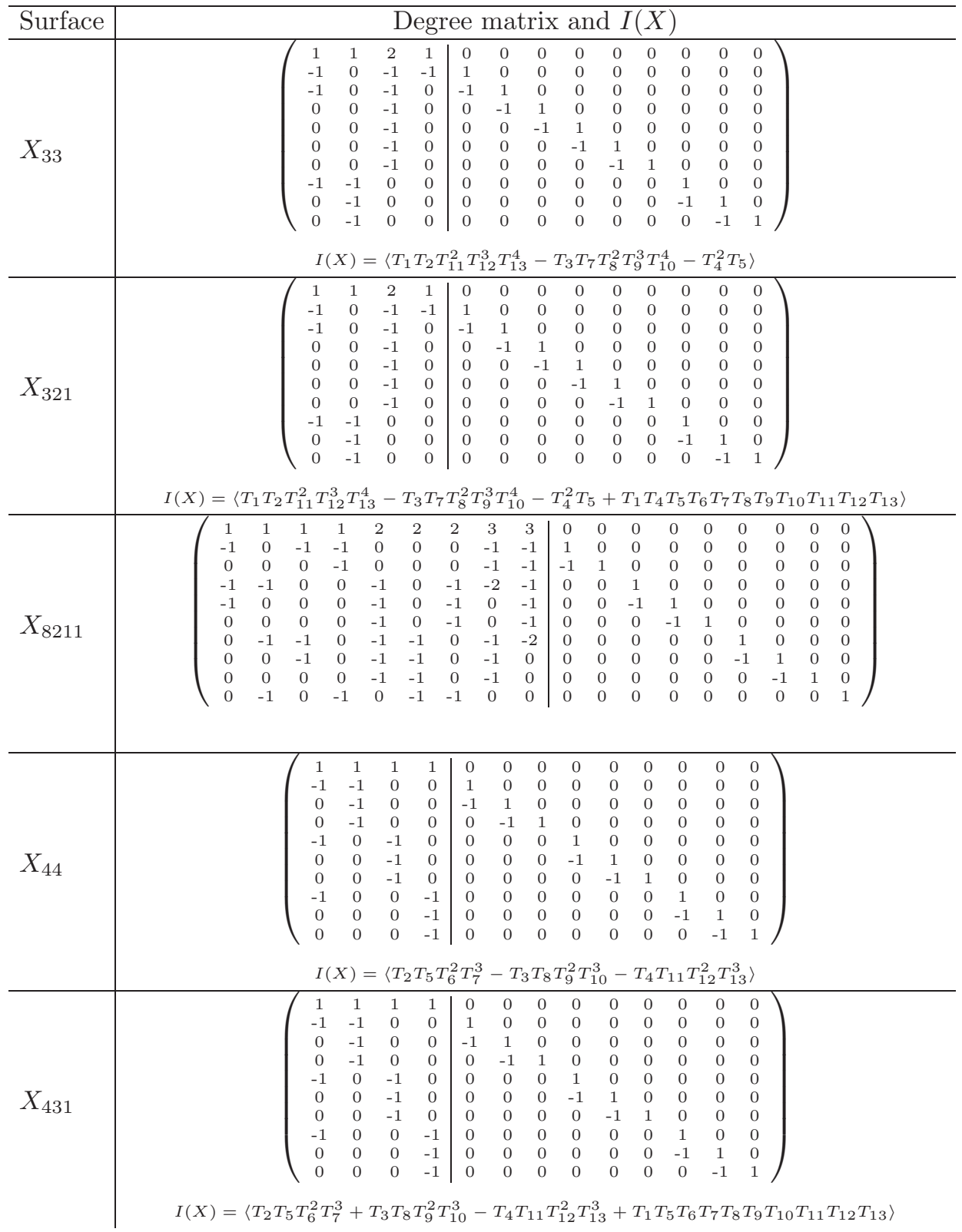


TABLE 5. (continued)

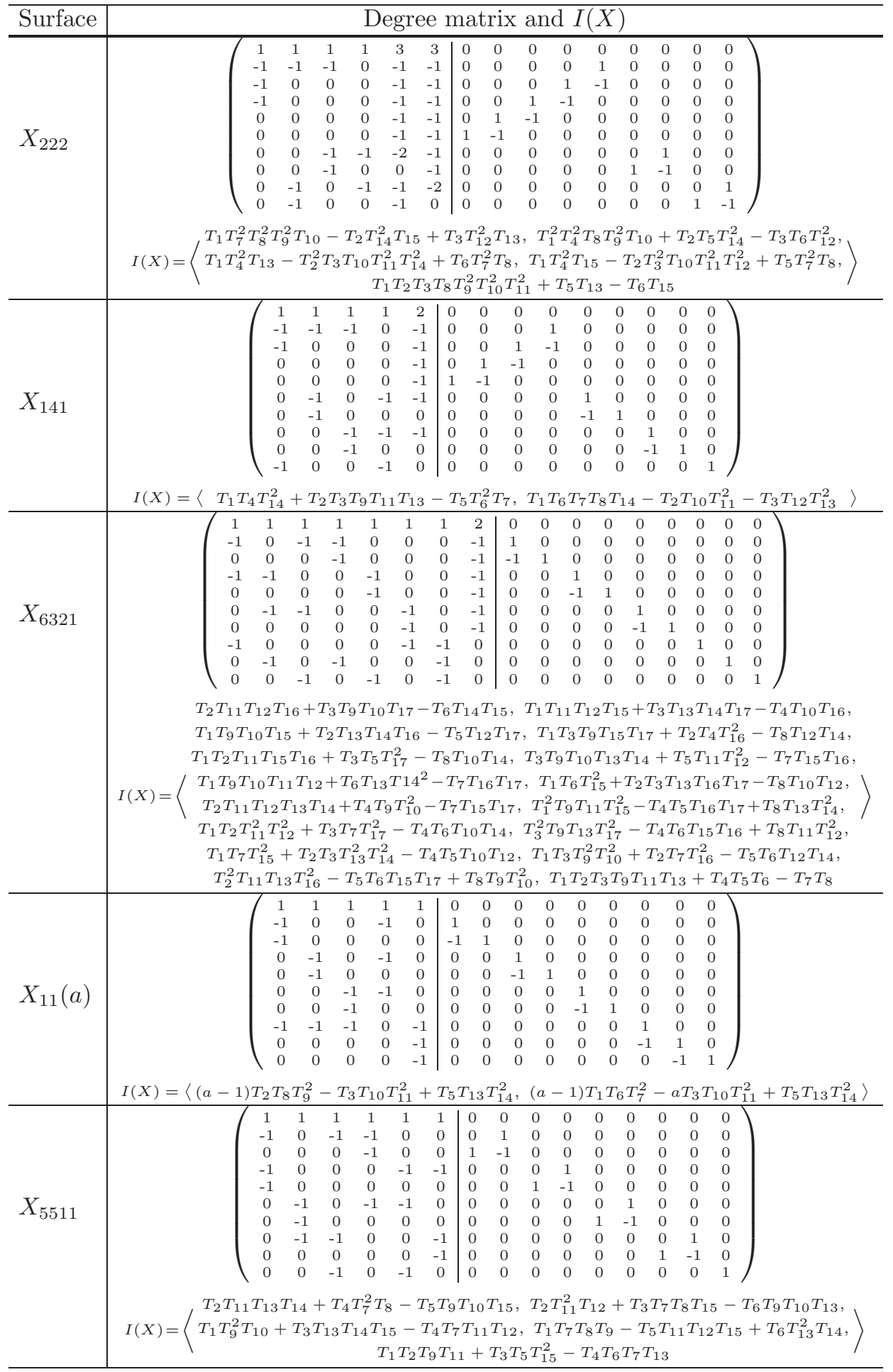


TABLE 5. (continued)

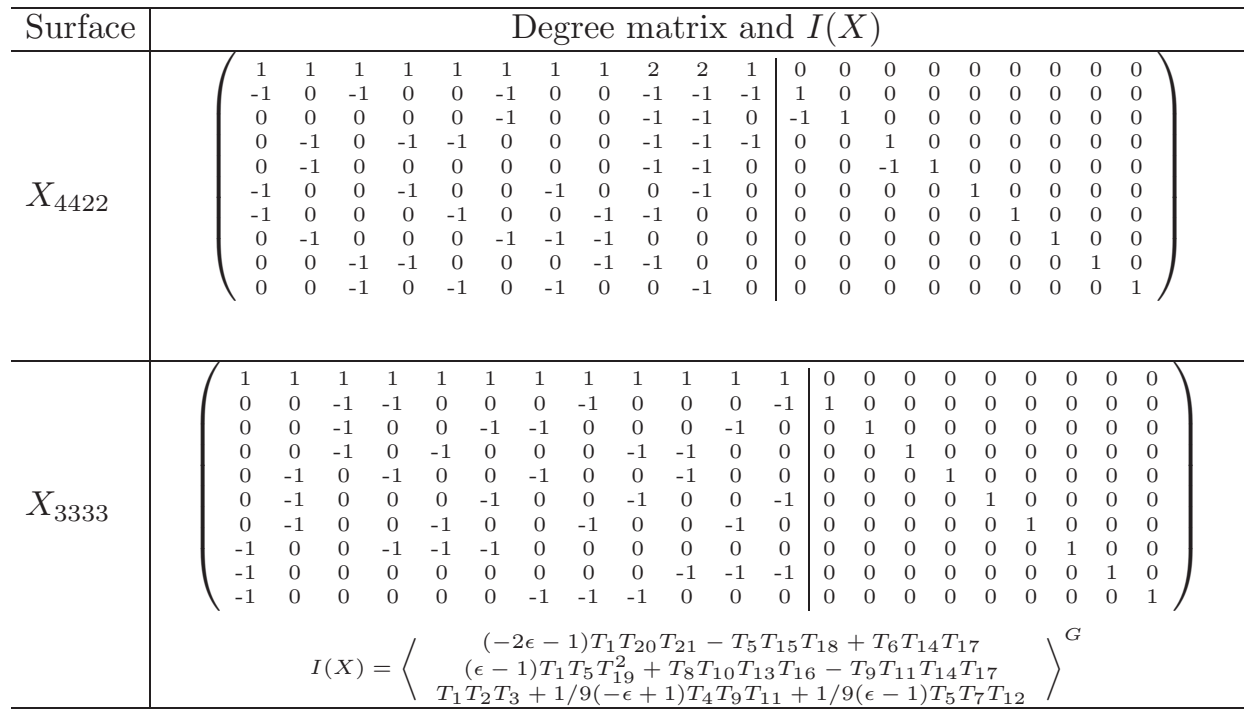

\section{ACKNOWLEDGMENT}

We thank Damiano Testa for several useful discussions.

\section{REFERENCES}

[AN06] Valery Alexeev and Viacheslav V. Nikulin, Del Pezzo and K3 surfaces, MSJ Memoirs, vol. 15, Mathematical Society of Japan, Tokyo, 2006. MR2227002 (2007e:14059)

[AD09] Michela Artebani and Igor Dolgachev, The Hesse pencil of plane cubic curves, Enseign. Math. (2) 55 (2009), no. 3-4, 235-273, DOI 10.4171/LEM/55-3-3. MR2583779 (2011d:14096)

[AL11] Michela Artebani and Antonio Laface, Cox rings of surfaces and the anticanonical Iitaka dimension, Adv. Math. 226 (2011), no. 6, 5252-5267, DOI 10.1016/j.aim.2011.01.007. MR2775900 (2012h:14012)

[ADHL15] Ivan Arzhantsev, Ulrich Derenthal, Jürgen Hausen, and Antonio Laface, Cox rings, Cambridge Studies in Advanced Mathematics, vol. 144, Cambridge University Press, Cambridge, 2015. MR3307753

[BHPVdV04] Wolf P. Barth, Klaus Hulek, Chris A. M. Peters, and Antonius Van de Ven, Compact complex surfaces, 2nd ed., Ergebnisse der Mathematik und ihrer Grenzgebiete. 3. Folge. [Results in Mathematics and Related Areas. 3rd Series], A Series of Modern Surveys in Mathematics, vol. 4, Springer-Verlag, Berlin, 2004. MR2030225 (2004m:14070)

[BHKS] Hendrik Bäker, Jürgen Hausen, and Keicherm Simon, On Chow quotients of torus actions, to appear in Michigan Math. J., available at http://arxiv.org/abs/1203. 3759.

[Bea83] Arnaud Beauville, Complex algebraic surfaces, London Mathematical Society Lecture Note Series, vol. 68, Cambridge University Press, Cambridge, 1983. Translated from the French by R. Barlow, N. I. Shepherd-Barron and M. Reid. MR732439 (85a:14024)

[BCP97] Wieb Bosma, John Cannon, and Catherine Playoust, The Magma algebra system. I. The user language, Computational algebra and number theory (London, 1993), J. Symbolic Comput. 24 (1997), no. 3-4, 235-265, DOI 10.1006/jsco.1996.0125. MR:1484478 
[Der14] Ulrich Derenthal, Singular del Pezzo surfaces whose universal torsors are hypersurfaces, Proc. Lond. Math. Soc. (3) 108 (2014), no. 3, 638-681, DOI 10.1112/plms/pdt041. MR3180592

[DGPS15] Wolfram Decker, Gert-Martin Greuel, Gerhard Pfister, and Hans Schönemann, SinGULAR 4-0-2 - A computer algebra system for polynomial computations (2015), available at http://www.singular.uni-kl.de.

[Dui10] Johannes J. Duistermaat, Discrete integrable systems, QRT maps and elliptic surfaces, Springer Monographs in Mathematics, Springer, New York, 2010. MR2683025 (2012g:37178)

[Har97] Brian Harbourne, Anticanonical rational surfaces, Trans. Amer. Math. Soc. 349 (1997), no. 3, 1191-1208, DOI 10.1090/S0002-9947-97-01722-4. MR.1373636 (97f:14007)

[Har77] Robin Hartshorne, Algebraic geometry, Graduate Texts in Mathematics, No. 52, Springer-Verlag, New York-Heidelberg, 1977. MR0463157 (57 \#3116)

[Hau13] Jürgen Hausen, Three lectures on Cox rings, Torsors, étale homotopy and applications to rational points, London Math. Soc. Lecture Note Ser., vol. 405, Cambridge Univ. Press, Cambridge, 2013, pp. 3-60. MR3077165

[HB03] D. Roger Heath-Brown, The density of rational points on Cayley's cubic surface, Proceedings of the Session in Analytic Number Theory and Diophantine Equations, Bonner Math. Schriften, vol. 360, Univ. Bonn, Bonn, 2003, pp. 33. MR2075628 (2005d:14033)

[Kod63] Kunihiko Kodaira, On compact analytic surfaces. II, III, Ann. of Math. (2) 77 (1963), 563-626; ibid. 78 (1963), 1-40. MR0184257 (32 \#1730)

[Mir89] Rick Miranda, The basic theory of elliptic surfaces, Dottorato di Ricerca in Matematica. [Doctorate in Mathematical Research], ETS Editrice, Pisa, 1989. MR:1078016 (92e:14032)

[MP86] Rick Miranda and Ulf Persson, On extremal rational elliptic surfaces, Math. Z. 193 (1986), no. 4, 537-558, DOI 10.1007/BF01160474. MR867347(88a:14044)

[Oka] Shinnosuke Okawa, On images of Mori dream spaces, to appear in Mathematische Annalen, DOI 10.1007/s00208-015-1245-5.

[Sal98] Per Salberger, Tamagawa measures on universal torsors and points of bounded height on Fano varieties, Nombre et répartition de points de hauteur bornée (Paris, 1996), Astérisque 251 (1998), 91-258. MR1679841(2000d:11091)

[SS10] Matthias Schütt and Tetsuji Shioda, Elliptic surfaces, Algebraic geometry in East Asia-Seoul 2008, Adv. Stud. Pure Math., vol. 60, Math. Soc. Japan, Tokyo, 2010, pp. 51-160. MR2732092(2012b:14069)

[Shi90] Tetsuji Shioda, On the Mordell-Weil lattices, Comment. Math. Univ. St. Paul. 39 (1990), no. 2, 211-240. MR 1081832 (91m:14056) CHILE

Departamento de Matemática, Universidad de Concepción, Casilla 160-C, Concepción,

E-mail address: martebani@udec.cl

Dipartimento di Matematica, Università Statale degli Studi di Milano via Saldini, 50, I-20133 Milano, Italy

E-mail address: alice.garbagnati@unimi.it

Departamento de Matemática, Universidad de Concepción, Casilla 160-C, Concepción, CHILE

E-mail address: alaface@udec.cl 